\title{
Contrasting the effects of suboptimally versus optimally presented affect primes on effort-related cardiac response
}

\author{
Ruta Lasauskaite Schüpbach • Guido H. E. Gendolla • \\ Nicolas Silvestrini
}

Published online: 30 September 2014

(C) Springer Science+Business Media New York 2014

\begin{abstract}
Participants worked on an easy versus difficult arithmetic task with integrated happiness versus sadness primes, presented either suboptimally (briefly and masked) or optimally (long and visible). As predicted by the IAPE model (Gendolla in International Journal of Psychophysiology 86:123-135, 2012. doi:10.1016/j.ijpsycho.2012.05.003), the affect primes moderated the task difficulty effect on mental effort in the suboptimal-prime condition: cardiac pre-ejection period response was stronger in the happiness/difficult than in the sadness/difficult condition and tended to be stronger in the sadness-easy than in the happiness-easy condition. These effects were reversed in the optimal-prime-presentation condition, suggesting behavior correction due to controlled prime processing. Moreover, neither suboptimally nor optimally presented affect primes had prime-congruent effects on conscious mood assessed via self-report. The results demonstrate differential effects of implicitly versus explicitly processed affect cues on mental effort and suggest that they can do so without inducing emotional feelings.
\end{abstract}

Keywords Cardiovascular reactivity - Implicit affect . Priming $\cdot$ Mental effort $\cdot$ IAPE model

\section{Introduction}

Enlarging to the literature on automaticity in behavior (see Bargh and Chartrand 1999; Dijksterhuis and Aarts 2010 for

R. Lasauskaite Schüpbach · G. H. E. Gendolla ( $₫)$.

N. Silvestrini

Geneva Motivation Lab, FPSE, Department of Psychology,

University of Geneva, 40, Bd. du Pont-d'Arve, 1211 Geneva 4,

Switzerland

e-mail: guido.gendolla@unige.ch reviews), the implicit-affect-primes-effort (IAPE) model (Gendolla 2012) posits that implicitly processed affective stimuli (i.e. affect primes) can influence effort mobilization through their effects on task demand experienced during performance. The IAPE model builds on the basic idea that effort mobilization follows a resource conservation principle. Consequently, the amount of mobilized effort is proportional to subjective demand as long as success is possible and justified, because people avoid mobilizing more resources than necessary (Brehm and Self 1989). In achievement contexts, people thus use all available information for evaluating task demand. According to the IAPE model, affect primes automatically activate knowledge about the respective affective states (i.e. implicit affect), including information about typically experienced performance ease or difficulty: Sadness and fear are associated with difficulty. Therefore sadness and fear primes lead to the experience of high demand and, as long as success is possible and justified, to higher effort. By contrast, happiness and anger are associated with performance ease and consequently lead to lower experienced demand.

A series of studies assessing effort as performance-related cardiovascular response has revealed support for the IAPE model. For moderately difficult tasks and "do-your-best" conditions, suboptimally ${ }^{1}$ presented sadness primes led to stronger effort-related cardiovascular response than both happiness and anger primes (Gendolla and Silvestrini 2011; Lasauskaite et al. 2013; Silvestrini and Gendolla 2011a). Supporting the IAPE model, participants in the sadness-prime condition also rated subjective task demand as higher than

\footnotetext{
${ }^{1}$ We use the term suboptimal rather than subliminal, because the latter refers to stimulus presentations below individually determined thresholds of conscious perception. In our experiments, low contrast affective stimuli are briefly presented $(27 \mathrm{~ms})$ and backward masked resulting in suboptimal presentation in order to prevent controlled processing of the primes' content.
} 
those in the happiness-prime condition. Moreover, affect primes moderated the effects of objective task difficulty (Freydefont et al. 2012; Silvestrini and Gendolla 2011b). When the task was easy, sadness-primes led to higher effort and higher perceived demand than happiness- and angerprimes. But when the task was difficult, sadness-primes led to high task demand but low effort because the task was perceived as too demanding compared to the happiness- and anger-prime conditions.

\section{Automatic versus controlled prime processing}

Experiments on implicit affect usually present affect primes suboptimally to give participants limited awareness of the primes' content in order to facilitate automatic responses and to prevent controlled reactions and the elicitation of fullblown emotions. Nevertheless, there has been considerable debate about the role of emotional feelings in affect priming (e.g., Clore et al. 2005; Winkielman et al. 2005; Winkielman and Schooler 2011). Therefore, Lasauskaite et al. (2013) aimed to test if suboptimally presented sadness- and happiness-primes have their effects on effort-related cardiovascular response because they induce emotional feelings. Half the participants were warned that "flickers" (i.e. primes) presented during the task could have an effect on their emotional feelings. It was expected that this warning should diminish the prime effect on effort-related cardiovascular response, if the primes induced feelings (e.g., Gendolla and Krüsken 2002). However, the prime effect in the warned condition remained and cardiac reactivity was even generally stronger, which was explained by increased cognitive load. Additionally, no evidence for prime effects on conscious feelings was found.

However, beside studies on affect primes, also other recent research on boundary conditions of automaticity is relevant for the present research question. There is evidence that doubt or inconfidence (DeMarree et al. 2012), warning of prime appearances (Verwijmeren et al. 2013), and instructions to attribute one's prime-induced thoughts to external cues (Loersch and Payne 2012) can moderate and reduce prime effects on behavior. In Study 2 by Loersch and Payne (2012), priming the concept of speed even produced a significant contrast effects (i.e. slower writing after priming the concept of speed) when participants were instructed to attribute their prime-induced thoughts to external stimuli. This suggest that controlled processing of primes and/or the concepts they make accessible can lead to behavior correction processes in terms of reduced prime assimilation and an increased probability of prime-contrast effects (cf. Herr 1986).

Suboptimal versus optimal affect prime presentation

Considering the above-discussed findings, the question arises what happens if affect primes are presented optimally and thus fully perceptible during task performance. One possibility is that fully visible affect primes elicit corresponding emotional feelings-emotion chronometry studies suggest that stimuli must be presented for at least $500 \mathrm{~ms}$ in order to elicit emotional reactions (see Gendolla 2012). Another possibility, related to the evidence discussed above, is that visible affect primes foster behavior correction.

Murphy and Zajonc (1993) found prime-assimilation effects on evaluative judgments of neutral targets only if affect primes were presented suboptimally (in this case even clearly subliminally: $4 \mathrm{~ms}$ ). In the optimal prime condition $(1,000 \mathrm{~ms})$, there was a trend to a prime-contrast effect in participants' judgments, suggesting controlled processing and correction of the prime influence (cf. Herr 1986). Rotteveel et al. (2001) found stronger affect prime effects on valence ratings of target stimuli and facial EMG responses when affect primes were presented suboptimally than when they were visible. Recently, Siegel and Weinberger (2012) found that repeated exposure to masked phobic stimuli reduced avoidance behavior and distress, whereas exposure to visible phobic stimuli increased distress without reducing avoidance behavior. This effect was still present after 1 year (Siegel and Warren 2013). These findings suggest that implicit processing of affective stimuli has different and sometimes stronger effects than explicit processing.

For effort mobilization, we see two alternatives for the effects of optimal affect prime presentation. (1) The first alternative suggests that optimally presented primes induce conscious affect and then have similar effects as suboptimal primes, but due to a different process. Conscious affect can be directly informative for evaluations of task demand and have mood-congruency effects on demand appraisals and effort mobilization: subjective demand is higher in a sad mood than in a happy mood (see Gendolla and Brinkmann 2005; Gendolla et al. 2012 for reviews). Such an effect would come close to the similar effects of implicit versus explicit self-awareness on effort mobilization (Silvia 2012). (2) The second alternative refers to behavior correction due to controlled prime processing. Accordingly, optimally presented primes should substantially reduce prime-assimilation and may even produce a primecontrast effect (e.g., Loersch and Payne 2012). The present study tested those alternatives.

\section{Effort-related cardiovascular response}

According to Wright's (1996) integration of motivational intensity theory (Brehm and Self 1989) with the active coping approach (Obrist 1981), effort is mirrored by betaadrenergic sympathetic nervous system impact on the heart. Among non-invasive measures, this is best reflected 
by increased cardiac contractility and thus shortened cardiac pre-ejection period (PEP)—-the time interval (in ms) between the onset of left ventricular excitation and the opening of the aortic valve (Berntson et al. 2004). Due to its impact on cardiac output (the volume of blood pumped by the ventricula per minute), contractility can also systematically influence systolic blood pressure (SBP) numerous previous studies have quantified effort as SBP because of this effect (see Gendolla and Richter 2010; Gendolla et al. 2012; Wright and Kirby 2001). However, PEP is the much more reliable and valid measure of effort mobilization, because it is directly influenced by betaadrenergic sympathetic impact (Kelsey 2012). SBP is additionally influenced by peripheral vascular resistance, which is not systematically influenced by beta-adrenergic activation (Levick 2003). Diastolic blood pressure (DBP) is even more strongly influenced by peripheral resistance. Heart rate (HR) is controlled by both sympathetic and parasympathetic influences and should reflect effort only to the degree of sympathetic activation (Berntson et al. 1993). Thus, in short, PEP is the most reliable effort indicator among these indices. Nevertheless, HR and blood pressure should always be assessed together with PEP in order to control for possible preload (ventricular filling) or afterload (arterial pressure) effects on PEP (Sherwood et al. 1990): Increased preload can decrease PEP due to higher ventricular filling and increased afterload can increase PEP because it takes longer to build up the necessary force to open the aortic valves.

The present study

We tested the effects of suboptimally versus optimally presented affect primes on effort-related cardiovascular response. Participants worked on an objectively easy or difficult arithmetic task during which they were exposed to suboptimally $(27 \mathrm{~ms})$ versus optimally $(780 \mathrm{~ms})$ presented sadness- or happiness-primes. (1) In the suboptimal-prime condition, we predicted the prime $\times$ difficulty crossover interaction effect on PEP reactivity we had found previously (Silvestrini and Gendolla 2011b). As depicted in Fig. 1, we anticipated (a) a weak PEP response in the happiness-prime/ easy and the sadness-prime/difficult conditions, and (b) a strong PEP response in the sadness-prime/easy and happinessprime/difficult conditions. This is predicted because, according to the IAPE model, suboptimally presented sadness-primes should lead to higher subjective demand during performance than happiness-primes in the easy condition. By contrast, in the objectively difficult condition, sadness-primes should lead to disengagement because the task is experienced as over-challenging while happiness-primes should lead to high but feasible demand. (2) In the optimal prime condition, where the primes were clearly visible, we expected (a) either

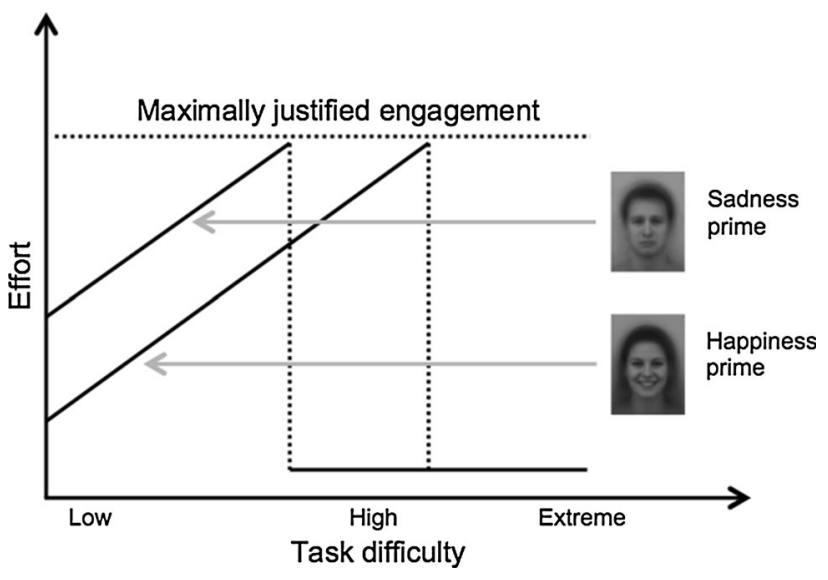

Fig. 1 Theoretical predictions for objective task difficulty effects on effort moderated by suboptimally presented sadness versus happiness primes. Averaged emotional facial expressions are prime examples from the AKDEF database (Lundqvist and Litton 1998). The figure is adapted from Silvestrini and Gendolla (2011b)

the same effects, but additional prime-related effects on conscious affect ratings, or (b) diminished (or even reversed) prime effects due to behavioral correction, as explained above.

\section{Methods}

Participants and design

One-hundred-and-thirty-four university students (127 women, average age 21 years) voluntarily and anonymously participated in the experiment for course credit or monetary remuneration (CHF 10, equivalent to USD 11) and were randomly assigned to a 2 (prime: sadness, happiness) $\times 2$ (task difficulty: easy, difficult) $\times 2$ (prime presentation: suboptimal, optimal) between-persons design. ${ }^{2}$

Affect prime stimuli

We used pictures of averaged neutral (FNES, MNES), sad (FSAS, MNAS), and happy (FHAS, MHAS) front perspective, low resolution, greyscale facial expressions taken from the Averaged Karolinska Directed Emotional Faces (AKDEF) database (Lundqvist and Litton 1998) as affect primes (see Fig. 1 for examples). Half the pictures showed male faces; half showed female faces.

\footnotetext{
${ }^{2}$ Due to technical measurement problems, there were missing data for some participants. Therefore, the sample sizes slightly varied across the analysis of the dependent variables: $N=134$ for PEP, $N=133$ for HR, and $N=130$ for SBP and DBP.
} 
Apparatus and physiological measures

To assess HR and cardiac PEP, impedance cardiogram (ICG) and electrocardiogram (ECG) signals were noninvasively assessed (sample rate $1,000 \mathrm{~Hz}$ ) with a Cardioscreen $^{\circledR} 1000$ system (medis, Ilmenau, Germany; see Scherhag et al. 2005 for a validation study). Four pairs of disposable spot electrodes were placed on the right and left sides of the base of the participant's neck and on the left and right middle axillary line at the height of the xiphoid.

We additionally assessed blood pressure with a Vaso$\operatorname{trac}^{\circledR}$ AMP205A monitor (MEDWAVE ${ }^{\circledR}$, St. Paul, MN, USA) that uses applanation tonometry with a pressure sensor placed on the wrist on top of the radial artery. Internal algorithms yield systolic and diastolic pressures approximately every $12-15$ heart beats, i.e. $4-5$ values/min (see Belani et al. 1999 for a validation study). All obtained cardiovascular measures were stored on internal drive and transferred to a personal computer. PEP, blood pressure, and HR values were calculated for 1-min intervals.

\section{Procedure}

The procedure was approved by the local ethical committee. After having obtained signed consent, the experimenter attached the electrodes and the blood pressure cuff and went to a control room. The procedure was computerized (E-Prime, Psychology Software Tools, Pittsburgh, PA, USA). Instructions were presented on the computer screen; responses were given with a numerical keyboard. At the beginning of the session, participants answered biographical questions and rated their current mood with 2 positive (joyful, cheerful) and 2 negative affect items (sad, depressed) of the Matthews et al. (1990) UWIST mood scale $(1=$ not at all to $7=$ very much $)$. Then participants watched a neutral documentary film showing landscapes (8 min) while physiological baseline measures were taken. This was followed by an arithmetic task (5 min) adapted from Bijleveld et al. (2010).

In the task, each trial presented an arithmetic equation, consisting of 3 added up single digits and a two-digit result (e.g., "7 $+5+3=14$ "). Participants had to decide for each equation if it was correct or not, by pressing a "yes" or a "no" key. Half of the presented equations were correct, half were incorrect. Participants received the instruction to try to respond correctly and as fast as possible. Each of the 36 trials started with a fixation cross (1,000 ms), followed by a facial expression picture (27 vs. $780 \mathrm{~ms}$ ) that was backward-masked with a noise picture showing scattered black and white dots (133 ms). Then the target equation appeared. Based on pretests, the maximal time response windows were 6,000 ms (easy task) versus 4,000 ms (difficult task). Participants' responses were followed by the feedback "Response entered" displayed for minimum $500 \mathrm{~ms}$. The inter-trial interval randomly varied between 2 and $5 \mathrm{~s}$.

Before the task, participants performed 10 training trials with neutral facial expressions as primes and received immediate feedback whether their answer was correct or not. No correctness feedback was given during the task to avoid possible affective reactions (e.g., Kreibig et al. 2012) that could interfere with the affect primes' impact. In the main task, emotional expressions were randomly displayed in $1 / 3$ of the trials. The remaining $2 / 3$ of the trials displayed neutral expressions. This priming procedure has been found to the most effective in the present paradigm (Silvestrini and Gendolla 2011a). Facial expression pictures were randomized in blocks of 6 ( 2 emotional, 4 neutral) and the same expression did not appear successively.

After the task, participants rated subjective task difficulty, mobilized effort, their capability to succeed, the importance and subjective value of success, their mathematical capacities, and how comfortable they felt in general with mental calculations $(1=$ not at all to $7=$ very $m u c h)$. The last two items were assessed to control for participants' arithmetic ability beliefs, which can systematically influence perceived task demand and effort (see Wright 1998; Wright and Kirby 2001). Finally, participants rated the same 4 mood adjectives as at the procedure's onset for assessing if the affect primes had an effect on their conscious affect. Then participants were debriefed, thanked, and received either course credit or the remuneration.

\section{Data analysis}

ICG signals were processed offline with software developed in our lab (Richter 2010). PEP (in ms) was determined as the interval between R-onset and B-point (Berntson et al. 2004). Shorter PEP indicated stronger cardiac contractility. B-point location was estimated based on the RZ interval of valid heart beat cycles (Lozano et al. 2007), visually inspected, and if necessary corrected as recommended (Sherwood et al. 1990).

With exception of the mood scores, data were analyzed with 2 (prime) $\times 2$ (difficulty) $\times 2$ (prime presentation) between-persons ANOVAs or ANCOVAs, respectively. Significant three-way interactions were decomposed with focused two-way interaction contrasts using the ANOVA $M S E$ in order to consider the total sample variance of the entire design for all statistical tests. Mood scores were analyzed with a 2 (prime) $\times 2$ (difficulty) $\times 2$ (prime presentation) $\times 2$ (time) mixed model ANOVA. The small number of men in our sample did not permit including gender as a separate factor. However, analyses ran without men led to basically the same results as those reported below. To facilitate interpretation of the results, effect sizes of $1 d f$ tests were transformed to eta-square. 
Ability can influence subjective task demand, effort-related cardiovascular response (see Wright 1998), and performance (Locke and Latham 1990). Consequently, we tested for possible associations between participants' arithmetic ability beliefs and both cardiovascular responses and task performance. The ratings of perceived capacity in mathematics and the self-evaluation of comfort with mental calculations were highly correlated and averaged to an ability-index $(r=.82$, $p<.001$; grand mean $M=3.53, S E=.15$ ). Preliminary ANCOVAs found significant associations between the ability-index and both response times (negative association), $F(1$, $125)=23.32, p<.001, \eta_{\mathrm{p}}^{2}=.16$, and accuracy scores (positive association), $F(1,125)=36.21, \quad p<.001$, $\eta_{\mathrm{p}}^{2}=.23$, in the arithmetic task. Therefore the ability-index was included as a covariate in the analysis of these task performance indices (see below). No associations emerged between the ability-index and any of the cardiovascular reactivity scores $(p s>.34)$. Consequently, the ability-index was not included as a covariate in these analyses.

\section{Results}

\section{Cardiovascular baselines}

Cardiovascular baseline scores for PEP, SBP, DBP, and $\mathrm{HR}$ were calculated as the averages of the last $5 \mathrm{~min}$ of the habituation period, which provided stable values (Cronbach's $\alpha$ s $>$.99). Cell means and standard errors appear in Table 1. Exploratory between-persons ANOVAs on these baseline scores found significant task difficulty main effects for SBP, $F(1,122)=18.28, p<.001, \eta_{\mathrm{p}}^{2}=.13$ (easy $M=120.77, \quad S E=1.66$, difficult $M=110.35$, $S E=1.79)$, and DBP, $F(1,122)=15.05, p<.001$, $\eta_{\mathrm{p}}^{2}=.11 \quad($ easy $\quad M=68.53, \quad S E=1.19, \quad$ difficult $M=61.72, S E=1.29$ ), while other effects were not significant ( $p s>.31$ ). The ANOVAs of PEP and HR baseline scores did not reveal any significant effects $(p s>.16)$.
Cardiovascular reactivity

Physiological reactivity scores were calculated for each participant by subtracting the baseline scores from the scores obtained during the task. As the 1-min change scores were highly consistent (Cronbach's as $>.95$ ), we created average reactivity scores for the entire task performance period. First, we tested for possible associations between baselines and reactivity scores with ANCOVAs in order to control for possible carryover or initial value effects (Llabre et al. 1991). No significant associations between cardiovascular baselines and reactivity scores emerged ( $p s>.13$ ). Thus, reactivity scores were analyzed without baseline adjustments.

We additionally tested for time effects during the task, by analyzing the 1-min reactivity scores with explorative 2 (prime) $\times 2$ (difficulty) $\times 2$ (prime presentation) $\times 5$ (time) mixed-model ANOVAs (with Greenhouse-Geisser corrections). These analyses revealed significant time main effects for all measures (all $p \mathrm{~s}<.015$ ) due to stronger general reactivity at the beginning of the task. However, except for SBP (see below) the reported effects were not further moderated by time ( $p s \geq .10)$.

\section{Cardiac PEP reactivity}

Cell means and standard errors are depicted in Fig. 2. A 2 (prime) $\times 2$ (difficulty) $\times 2$ (prime presentation) ANOVA of PEP reactivity revealed a significant three-way interaction, $F(1$, 126) $=8.46, p=.004, \eta_{\mathrm{p}}^{2}=.06$. To decompose this interaction, we ran focused crossover interaction contrasts which showed significant prime $\times$ difficulty interactions in both the suboptimal, $F(1,126)=4.36, p=.039, \eta_{\mathrm{p}}^{2}=.03$, and the optimal-prime condition, $F(1,126)=4.08, p=.045$, $\eta_{\mathrm{p}}^{2}=.03$. As visible in Fig. 2, the pattern of PEP reactivity in the suboptimal-prime condition corresponded to our prediction about the moderation of objective task difficulty effects by the primes. Most relevant for the present research, this pattern was reversed in the optimal prime condition.

Table 1 Cell means and standard errors (in parentheses) for cardiovascular baseline values

\begin{tabular}{|c|c|c|c|c|c|c|c|c|}
\hline & \multicolumn{4}{|c|}{ Suboptimal prime } & \multicolumn{4}{|l|}{ Optimal prime } \\
\hline & \multicolumn{2}{|l|}{ Easy task } & \multicolumn{2}{|l|}{ Difficult task } & \multicolumn{2}{|l|}{ Easy task } & \multicolumn{2}{|l|}{ Difficult task } \\
\hline & $\begin{array}{l}\text { Happiness } \\
\text { prime }\end{array}$ & $\begin{array}{l}\text { Sadness } \\
\text { prime }\end{array}$ & $\begin{array}{l}\text { Happiness } \\
\text { prime }\end{array}$ & $\begin{array}{l}\text { Sadness } \\
\text { prime }\end{array}$ & $\begin{array}{l}\text { Happiness } \\
\text { prime }\end{array}$ & $\begin{array}{l}\text { Sadness } \\
\text { prime }\end{array}$ & $\begin{array}{l}\text { Happiness } \\
\text { prime }\end{array}$ & $\begin{array}{l}\text { Sadness } \\
\text { prime }\end{array}$ \\
\hline PEP & $97.08(3.68)$ & $96.19(2.61)$ & $99.31(2.95)$ & $102.30(2.75)$ & $95.83(3.31)$ & $100.32(2.66)$ & $101.08(2.70)$ & $98.12(1.89)$ \\
\hline SBP & $124.27(4.08)$ & $118.24(5.20)$ & $110.08(1.88)$ & $109.76(2.49)$ & $119.18(3.51)$ & $121.38(3.21)$ & $111.71(1.74)$ & $109.83(2.54)$ \\
\hline DBP & $70.21(2.55)$ & $67.74(3.90)$ & $61.26(1.56)$ & $62.19(1.88)$ & $67.25(2.36)$ & $68.92(2.53)$ & $62.32(1.49)$ & $61.12(1.61)$ \\
\hline HR & $76.39(2.52)$ & $75.73(2.81)$ & $79.72(3.41)$ & $79.08(2.97)$ & 77.38 (2.63) & 76.95 (2.91) & $74.19(2.93)$ & $76.96(1.96)$ \\
\hline
\end{tabular}

Cell $n$ s ranging from 15 to 19

$P E P$ pre-ejection period, $S B P$ systolic blood pressure, $D B P$ diastolic blood pressure, $H R$ heart rate. Units of measures are milliseconds for PEP, millimeters of mercury for SBP and DBP, and beats per minute for HR 
Fig. 2 PEP reactivity scores $( \pm$ SEM $)$ in the experimental conditions



Additional cell contrasts revealed that the sadness-prime and happiness-prime cells differed significantly from one another when the task was difficult in both the suboptimal, $t(126)=2.16, p=.033, \eta^{2}=.04$, and optimal, $t(126)=2.48, p=.015, \eta^{2}=.05$, prime presentation conditions. However, the directions of the effects were opposite: In the suboptimal-prime condition, reactivity in the happinessprime/difficult condition $(M=-2.95, S E=1.20)$ was stronger than in the sadness-prime/difficult condition $(M=.23, S E=1.54)$. By contrast, in the optimal-prime condition, PEP reactivity in the sadness-prime/difficult condition $(M=-2.16, S E=.62)$ was stronger than in the happiness-prime/difficult cell $(M=1.49, S E=1.09)$. The cell differences between sadness- and happiness-prime cells were not significant in the easy condition (both $p s>.45$; suboptimal presentation: happiness-prime $M=-1.98, S E=.70$, sadness-prime $M=-3.00, S E=.98$; optimal presentation: happiness-prime $M=-2.00, \quad S E=.94, \quad$ sadness-prime $M=-1.59, S E=.67)$. Moreover, also PEP responses in the happiness-prime/difficult condition clearly differed between the suboptimal and optimal prime conditions, as evident in a significant cell contrast, $t(126)=3.02, p=.003, \eta^{2}=.07$.

\section{$S B P$ reactivity}

Cell means and standard errors appear in Table 2. The 2 (prime) $\times 2$ (difficulty) $\times 2$ (prime presentation) ANOVA of the SBP responses revealed a significant task difficulty $\times$ prime presentation interaction, $F(1,122)=5.06$, $p=.026, \eta_{\mathrm{p}}^{2}=.04$. While the difference between the easy $(M=4.57, S E=1.23)$ and difficult $(M=5.21, S E=.71)$ conditions was not significant in the suboptimal-prime condition ( $p=.602)$, SBP response was stronger in the difficult $(M=6.52, S E=.83)$ than in the easy condition $(M=1.55, \quad S E=.85), \quad F(1, \quad 122)=13.73, \quad p<.001$, $\eta^{2}=.10$, in the optimal prime-condition. The three-way interaction on SBP reactivity was not significant $(p=.210)$, although the pattern of cell means corresponded to the effects of cardiac PEP.

However, an explorative $2 \times 2 \times 2 \times 5$ mixed-model ANOVA revealed a significant four-way interaction, $F(4$, $488)=2.51, p=.049, \eta_{\mathrm{p}}^{2}=.02$. Follow-up tests found a significant three-way interaction effect on systolic reactivity during the first minute, $F(1,122)=5.04, p=.027$, $\eta_{\mathrm{p}}^{2}=.04$, but not during the following minutes $(p \mathrm{~s}>.29)$. The interaction contrast for the first minute was not significant in the optimal-prime condition $(p=.205)$ and only trended towards significance in the suboptimal prime condition, $F(1,122)=3.57, p=.06, \eta^{2}=.03$. According to the cell means, which appear in Table 2, the pattern was largely compatible with that of PEP during the task, but less pronounced.

\section{$D B P$ reactivity}

A 2 (prime) $\times 2$ (difficulty) $\times 2$ (prime presentation) ANOVA of DBP reactivity (see Table 2 ) found effects that corresponded to those of SBP. The task difficulty $\times$ prime presentation interaction was significant, $F(1,122)=4.06$, $p=.046, \eta_{\mathrm{p}}^{2}=.03$, while the three-way interaction was not $(p=.205)$. Again, the difference between the easy $(M=3.27, S E=.89)$ and difficult $(M=3.57, S E=.51)$ conditions was not significant in the suboptimal-prime condition $(p=.723)$. But in the optimal-prime condition, DBP 
Table 2 Cell means and standard errors (in parentheses) of the blood pressure and heart rate reactivity scores during task performance

\begin{tabular}{|c|c|c|c|c|c|c|c|c|}
\hline & \multicolumn{4}{|c|}{ Suboptimal prime } & \multicolumn{4}{|c|}{ Optimal prime } \\
\hline & \multicolumn{2}{|l|}{ Easy task } & \multicolumn{2}{|c|}{ Difficult task } & \multicolumn{2}{|l|}{ Easy task } & \multicolumn{2}{|c|}{ Difficult task } \\
\hline & $\begin{array}{l}\text { Happiness } \\
\text { prime }\end{array}$ & $\begin{array}{l}\text { Sadness } \\
\text { prime }\end{array}$ & $\begin{array}{l}\text { Happiness } \\
\text { prime }\end{array}$ & $\begin{array}{l}\text { Sadness } \\
\text { prime }\end{array}$ & $\begin{array}{l}\text { Happiness } \\
\text { prime }\end{array}$ & $\begin{array}{l}\text { Sadness } \\
\text { prime }\end{array}$ & $\begin{array}{l}\text { Happiness } \\
\text { prime }\end{array}$ & $\begin{array}{l}\text { Sadness } \\
\text { prime }\end{array}$ \\
\hline SBP & $2.51(1.40)$ & $6.52(1.93)$ & $5.79(1.09)$ & $4.63(.91)$ & $.65(1.08)$ & $2.50(1.32)$ & $5.80(1.42)$ & $7.23(.87)$ \\
\hline SBP 1st min & $4.22(1.51)$ & $7.90(2.04)$ & $9.08(1.52)$ & $6.96(.85)$ & $2.57(1.32)$ & $1.71(1.64)$ & $8.42(1.66)$ & 11.45 \\
\hline DBP & $1.85(.91)$ & $4.62(1.47)$ & $3.91(.77)$ & $3.23(.69)$ & $.80(.68)$ & $1.56(.93)$ & $3.85(.95)$ & $4.58(.66)$ \\
\hline HR & $2.74(.66)$ & $4.15(.97)$ & $2.49(.71)$ & $3.23(1.24)$ & $2.92(1.00)$ & $1.94(.83)$ & $3.18(1.12)$ & $3.00(1.03)$ \\
\hline
\end{tabular}

Cell $n$ s ranging from 15 to 19 . Units of measures are millimeters of mercury for SBP and DBP and beats per minute for HR

reactivity was stronger in the difficult $(M=4.21, S E=.57)$ than in the easy condition $(M=1.17, S E=.57)$, $t(122)=3.20, p=.002, \eta^{2}=.08$

\section{$H R$ reactivity}

A 2 (prime) $\times 2$ (difficulty) $\times 2$ (prime presentation) ANOVA of HR reactivity (see Table 2) did not find any effects $(p s>.39)$.

Task performance and self-report measures

\section{Performance}

Two (prime) $\times 2$ (difficulty) $\times 2$ (prime presentation) ANOVAs of ability-index-adjusted task performance measures did not find any effects, neither on response times for correct responses (grand $M=2,712 \mathrm{~ms}, \quad S E=23.25$; $p s>.40$ ) nor on accuracy scores (grand $M=73 \%$, $S E=1.07 ; p s>.10)$.

\section{Mood}

Cell means appear in Table 3. Given the high inter-correlations of the ratings of positive and inversed negative affect UWIST scale items, we created mood sum scores for the pretask (Cronbach's $\alpha=.76$ ) and post-task (Cronbach's $\alpha=.71)$ mood measures. A 2 (prime) $\times 2$ (difficulty) $\times 2$ (prime presentation) $\times 2$ (time) mixed-model ANOVA revealed a significant time main effect, $F(1,126)=112.60$, $p<.001, \eta_{\mathrm{p}}^{2}=.47$ (pre-task $M=21.07, S E=.34$, posttask $M=16.65, S E=.40)$, which was qualified by a significant four-way interaction, $F(1,126)=8.47, p=.004$, $\eta_{\mathrm{p}}^{2}=.06$, in absence of other significant effects $(p \mathrm{~s}>.10)$. We decomposed the four-way interaction with separate 2 (prime) $\times 2$ (difficulty) $\times 2$ (prime presentation) ANOVAs of the pre-task and post-task mood scores. The three-way interaction was not significant for the pre-task mood scores $(p=.509)$, but for the post-task scores, $F(1,120)=5.99$, $p=.016, \eta_{\mathrm{p}}^{2}=.05$. Focused interaction contrasts revealed a significant prime $\times$ difficulty interaction in the suboptimalprime condition $F(1,126)=5.23, p=.024, \eta_{\mathrm{p}}^{2}=.04$, but not in the optimal-prime condition $(p=.243)$. Additional cell contrasts between the sadness-prime and happiness-prime cells in the suboptimal-prime condition revealed no significant difference when the task was easy $(p=.456)$, but a significant effect when the task was difficult, $t(126)=2.43$, $p=.017, \eta_{\mathrm{p}}^{2}=.06$. Here, the mood scores in the happinessprime cell were lower than in the sadness-prime cell. In the optimal-prime condition, the contrasts did neither find significant differences between the sadness-prime and happiness-prime cells in the easy ( $p=.641)$ nor in the difficult condition ( $p=.246$ ). Moreover, the prime main effect in the three-way ANOVA was not significant $(p=.605)$. These findings do not provide any evidence for the possibility that the affect primes influenced participants' conscious moods in a prime-congruent way.

\section{Task ratings}

Cell means are presented in Table 3 . The ratings of subjective difficulty and capability were negatively correlated, $r=-.51$, and the ratings of importance and value of success were positively correlated, $r=.60$ (both $p \mathrm{~s}<.01$ ). To reduce the number of statistical tests we thus created a demand score (average of difficulty and reverse-coded capability) and a value of success score (average of success value and importance). A 2 (prime) $\times 2$ (difficulty) $\times 2$ (prime presentation) ANOVA of the demand score revealed a three-way interaction as the only significant effect, $F(1,126)=7.34, p=.008$, $\eta_{\mathrm{p}}^{2}=.06$ (other $\left.p \mathrm{~s}>.10\right)$. The prime $\times$ difficulty interaction contrast was not significant in the optimal-prime condition $(p=.30)$, but in the suboptimal-prime condition, $F(1$, $126)=7.78, p=.006, \eta_{p}^{2}=.06$. In the easy condition, subjective demand did not differ significantly between the happiness-prime and sadness-prime conditions ( $p=.238)$. In the difficult condition, ratings were significantly higher in the happiness-prime than in the sadness-prime condition, 
Table 3 Cell means and standard errors (in parentheses) for self-report measures

\begin{tabular}{|c|c|c|c|c|c|c|c|c|}
\hline & \multicolumn{4}{|c|}{ Suboptimal prime } & \multicolumn{4}{|c|}{ Optimal prime } \\
\hline & \multicolumn{2}{|l|}{ Easy task } & \multicolumn{2}{|l|}{ Difficult task } & \multicolumn{2}{|l|}{ Easy task } & \multicolumn{2}{|l|}{ Difficult task } \\
\hline & $\begin{array}{l}\text { Happiness } \\
\text { prime }\end{array}$ & $\begin{array}{l}\text { Sadness } \\
\text { prime }\end{array}$ & $\begin{array}{l}\text { Happiness } \\
\text { prime }\end{array}$ & $\begin{array}{l}\text { Sadness } \\
\text { prime }\end{array}$ & $\begin{array}{l}\text { Happiness } \\
\text { prime }\end{array}$ & $\begin{array}{l}\text { Sadness } \\
\text { prime }\end{array}$ & $\begin{array}{l}\text { Happiness } \\
\text { prime }\end{array}$ & $\begin{array}{l}\text { Sadness } \\
\text { prime }\end{array}$ \\
\hline Pre-task mood & $20.47(.84)$ & $20.79(1.11)$ & $20.93(.80)$ & 20.81 (1.09) & $22.39(.87)$ & $21.94(.93)$ & $20.13(1.00)$ & $21.07(1.03)$ \\
\hline Post-task mood & $18.00(1.12)$ & $16.84(1.14)$ & $14.33(1.52)$ & $18.38(.96)$ & $17.56(1.12)$ & $18.28(.95)$ & $15.88(1.13)$ & 13.93 \\
\hline Demand & $3.32(.25)$ & $3.82(.27)$ & $4.33(.33)$ & $3.13(.27)$ & $3.61(.34)$ & $3.50(.31)$ & $3.85(.36)$ & $4.37(.29)$ \\
\hline Subjective effort & $4.18(.35)$ & $4.74(.37)$ & $4.53(.29)$ & $3.88(.26)$ & $4.61(.34)$ & $4.78(.35)$ & $4.69(.28)$ & $5.00(.38)$ \\
\hline Success value & $5.59(.13)$ & $5.63(.18)$ & $4.67(.38)$ & $4.69(.34)$ & $5.56(.23)$ & $5.78(.19)$ & $4.97(.32)$ & $5.10(.24)$ \\
\hline
\end{tabular}

Cell $n$ s ranging from 15 to 19 . Sum scores for mood and mean scores (ranging from 1 to 7 ) for the subjective demand and value of success indices and the subjective effort ratings

$t(126)=2.71, p=.008, \eta^{2}=.04$. No significant effects emerged on the effort ratings ( $p s \geq .066$ ), though the pattern of cell means largely corresponds to that of PEP.

The ANOVA of the value of success index revealed only a surprising main effect of the task difficulty manipulation, $F(1,126)=18.78, p<.001, \eta_{\mathrm{p}}^{2}=.13$, due to higher scores in the easy $(M=5.64, S E=.09)$ than in the difficult condition $(M=4.86, S E=.16)$ (other $p \mathrm{~s}>.25)$.

\section{Discussion}

The present study provides first evidence for differential effects of implicit versus explicit affect primes on effortrelated cardiac response, with a prime assimilation effect on effort when the primes were presented suboptimally, but a prime contrast effect when the primes were clearly visible.

Suboptimally presented sadness and happiness cues moderated the effect of objective task difficulty on cardiac PEP response as predicted by the IAPE model (Gendolla 2012) and found in previous studies (Freydefont et al. 2012; Silvestrini and Gendolla 2011b). Accordingly, in the difficult condition, the PEP response was stronger in the happiness-prime than in the sadness-prime condition. The IAPE model predicts this, because of additive effect of objective task difficulty and prime-induced accessibility of the difficulty (sadness condition) and ease (happiness condition) concepts on experienced demand during performance. This leads to high effort in the happiness-prime/ difficult (high, but possible demand) and low effort in the sadness-prime/difficult condition (disengagement due to excessive demand). In the easy condition, the prime effect should be reversed-low effort due to low demand in the happiness-prime condition, but high effort due to high but feasible demand in the sadness-prime condition. The prime $\times$ difficulty crossover interaction contrast was significant and the PEP response pattern largely corresponded to our hypothesis for the suboptimal prime condition, but cell contrasts revealed that the prime effect was only significant in the difficult condition. For the present study, the lack of a significant prime effect in the easy conditionwhere our previous studies had found significant prime effects-can be explained with too low objective task difficulty, leaving little room for the affect primes to influence subjective demand during performance. Our arithmetic task was new-the original version (Bijleveld et al. 2010) was not difficulty-manipulated. Apparently, our easy version was easier than intended.

Most relevant, when the affect primes were clearly visible, they also moderated the effect of objective task difficulty on PEP, but the direction of this effect was opposite to the suboptimal prime condition. This is interpretable as a prime contrast effect on effort-related cardiac response when the primes were clearly visible, reflecting the result of a shift from automatic (suboptimal presentation) to controlled (optimal presentation) processing (cf. Bijleveld et al. 2012). The results speak for a behavior correction process in the optimal-prime condition-which is only possible for conscious processes (Bijleveld et al. 2012; Dehaene and Naccache 2001). The effect resembles those of inconfidence (DeMarree et al. 2012), warnings of prime appearance (Verwijmeren et al. 2013), or instructions to control prime-induced thoughts (Loersch and Payne 2012) demonstrated in other studies. We suspect that the deeper motivational reason for such behavior correction effects may rely in psychological reactance (Brehm 1966). If people prefer autonomy and basically think that they act in accordance with their own decisions, they should dislike being manipulated (Ryan and Deci 2000). Visible emotional expressions, which have nothing to do with the task itself-and which were not present during the training trials where only visible neutral expressions appearedshould elicit suspicion (cf. DeMarree et al. 2012) that one is manipulated, leading to behavior correction. The effect of affect primes on effort is more indirect than the effect of 
behavior primes on corresponding actions (see Gendolla and Silvestrini 2015) and thus more difficult to be corrected. But if also the clearly visible affect primes elicit a spontaneous tendency to mobilize more (sadness) or less (happiness) effort, a reaction against the feeling to be manipulated could be doing the opposite. However, we have to leave it for future research to further test this idea.

Among our cardiovascular measures only PEP reactivity-the most sensitive noninvasive index of beta-adrenergic sympathetic impact on the heart (see Kelsey 2012) showed the significant three-way interaction effect of affect primes, task difficulty, and prime presentation for the entire task. The patterns of SBP and DBP responses were compatible with our effort-related predictions and the PEP effects, but the three-way interaction was not significant there. SBP reactivity during the first minute of the task largely resembled that of PEP, but was less pronounced. These discrepancies are not surprising because PEP is the purest index of beta-adrenergic sympathetic impact among the assessed cardiovascular activity indices and the prime measure of effort (Kelsey 2012; Obrist 1981; Wright 1996), although cardiac contractility has also a systematic impact on SBP by influencing cardiac output (Richter et al. 2008). Also the lack of effects on HR is not problematic. $\mathrm{HR}$ is determined by the independent influences of the sympathetic and parasympathetic nervous systems and should only reflect effort mobilization to the degree to which HR is determined by sympathetic arousal rather than parasympathetic withdrawal. Most relevant, we have not found evidence for PEP effects and simultaneous decreases in blood pressure or HR. Therefore the present PEP effects can hardly be explained by pre- or afterload effects, allowing the conclusion that they reflect beta-adrenergic impact (see Sherwood et al. 1990).

The present effects on the measures of subjective demand assessed after the task only partially corresponded to the pattern one would anticipate according to the IAPE model (Gendolla 2012). The lower demand ratings in the happiness-prime/easy condition than in the sadness-prime/ easy condition are what one would expect. But when the task was difficult, one should again expect higher demand ratings in the sadness-prime condition than in the happiness-prime condition. Surprisingly, those ratings were reversed. In the optimal-prime condition effects were not significant. However, it is important to note that the IAPE model posits that affect primes have a systematic impact on experienced task demand during task performance, i.e. when effort is mobilized. Given that this is barely possible in our experimental paradigm, demand-related ratings were assessed after performance-though retrospective judgments can suffer from a number of biases (see Robinson and Clore 2002). Given our previous evidence for affect primes' effects on subjective demand (Gendolla and
Silvestrini 2011; Lasauskaite et al. 2013; Silvestrini and Gendolla 2011b), we can only attribute the effect in the suboptimal-prime/difficult condition to chance. The same applies to the surprising difficulty effect on the retrospective value of success ratings.

However, we might also assume that task demand is evaluated automatically, i.e. without awareness (De Houwer et al. 2009). This way, the discrepancy between effortrelated cardiac response and the demand ratings would not be surprising because self-reports only measure what can be consciously perceived and reflected. Accordingly, subjective demand during the task and self-reports of perceived demand measured after the task assess different issues-which may also explain that subjective effort ratings after the task were not significantly influenced by the manipulation, though the pattern of cell means resembles that of PEP and also SBP response in the first task minute.

Our measures of response accuracy and reaction times did not reveal significant effects. Although some researchers consider speed and accuracy as indicators of effort (e.g., Bijleveld et al. 2010) and some of our lab's previous studies found performance effects that corresponded to effort-related cardiovascular response (e.g., Gendolla and Silvestrini 2010, 2011; Lasauskaite et al. 2013), we had not predicted any performance effects, because effort and performance can be dissociated. Performance depends on more than effort-at least ability and strategy use are important additional factors (Locke and Latham 1990). Thus, lacking performance effects are hardly surprising.

Finally, our mood measures did not provide any evidence that the happiness and sadness primes-neither optimal nor suboptimal-induced prime-congruent happiness- or sadness-related feeling states, which corresponds to our previous studies (Freydefont and Gendolla 2012; Freydefont et al. 2012; Gendolla and Silvestrini 2011; Lasauskaite et al. 2013; Silvestrini and Gendolla 2011a, b). Although zero-effects do not permit firm conclusions, this lack of evidence for prime effects on conscious affect goes along with one of the main ideas of the IAPE model: It is not necessary that affect primes induce conscious affect to influence effort. It is sufficient that the primes make emotion knowledge about typical performance ease or difficulty accessible, which then influences subjective task demand and corresponding effort during performance. The present findings suggest that clearly visible primes lead to prime-contrast effects on mental effort, which are typical for controlled prime processing.

Acknowledgments This research was supported by a research grant from the Swiss National Science Foundation awarded to the second author (SNF 100014-131760/1). We thank Audrey Bridy for her help as a hired experimenter. 


\section{References}

Bargh, J. A., \& Chartrand, T. L. (1999). The unbearable automaticity of being. American Psychologist, 54, 462-479. doi:10.1037/ 0003-066X.54.7.462.

Belani, K. G., Buckley, J. J., \& Poliac, M. O. (1999). Accuracy of radial artery blood pressure determination with the Vasotrac ${ }^{\mathrm{TM}}$. Canadian Journal of Anesthesia/Journal Canadien d'Anesthésie, 46, 488-496. doi:10.1007/BF03012951.

Berntson, G. G., Cacioppo, J. T., \& Quigley, K. S. (1993). Cardiac psychophysiology and autonomic space in humans: Empirical perspectives and conceptual implications. Psychological Bulletin, 114, 296-322. doi:10.1037/0033-2909.114.2.296.

Berntson, G. G., Lozano, D. L., Chen, Y.-J., \& Cacioppo, J. T. (2004). Where to Q in PEP. Psychophysiology, 41, 333-337. doi:10. 1111/j.1469-8986.2004.00156.x.

Bijleveld, E., Custers, R., \& Aarts, H. (2010). Unconscious reward cues increase invested effort, but do not change speed-accuracy tradeoffs. Cognition, 115, 330-335. doi:10.1016/j.cognition. 2009.12.012

Bijleveld, E., Custers, R., \& Aarts, H. (2012). Human reward pursuit: From rudimentary to higher-level functions. Current Directions in Psychological Science, 21, 194-199. doi:10.1177/ 096372141243846.

Brehm, J. W. (1966). A theory of psychological reactance. New York: Academic Press.

Brehm, J. W., \& Self, E. A. (1989). The intensity of motivation. Annual Review of Psychology, 40, 109-131. doi:10.1146/ annurev.ps.40.020189.000545.

Clore, G. L., Storbeck, J., Robinson, M. D., \& Centerbar, D. B. (2005). Seven sins in the study of unconscious affect. In L. F. Barrett, P. M. Niedenthal, \& P. Winkielman (Eds.), Emotion and consciousness (pp. 384-408). New York: Guilford.

De Houwer, J., Teige-Mocigemba, S., Spruyt, A., \& Moors, A. (2009). Implicit measures: A normative analysis and review. Psychological Bulletin, 135, 347-368. doi:10.1037/a0014211.

Dehaene, S., \& Naccache, L. (2001). Towards a cognitive neuroscience of consciousness: Basic evidence and a workspace framework. Cognition, 79, 1-37. doi:10.1016/S0010-0277(00)00123-2.

DeMarree, K. G., Loersch, C., Briñol, P., Petty, R. E., Payne, B. K., \& Rucker, D. D. (2012). From primed construct to motivated behavior: Validation processes in goal pursuit. Personality and Social Psychology Bulletin, 38, 1659-1670. doi:10.1177/ 0146167212458328.

Dijksterhuis, A., \& Aarts, H. (2010). Goals, attention, and consciousness. Annual Review of Psychology, 61, 461-490. doi:10.1146/ annurev.psych.093008.100445.

Freydefont, L., \& Gendolla, G. H. E. (2012). Incentive moderates the impact of implicit anger versus sadness cues on effort-related cardiac response. Biological Psychology, 91, 120-127. doi:10. 1016/j.biopsycho.2012.04.002.

Freydefont, L., Gendolla, G. H. E., \& Silvestrini, N. (2012). Beyond valence: The differential effect of masked anger and sadness stimuli on effort-related cardiac response. Psychophysiology, 49, 665-671. doi:10.1111/j.1469-8986.2011.01340.x.

Gendolla, G. H. E. (2012). Implicit affect primes effort: A theory and research on cardiovascular response. International Journal of Psychophysiology, 86, 123-135. doi:10.1016/j.ijpsycho.2012.05. 003.

Gendolla, G. H. E., \& Brinkmann, K. (2005). The role of mood states in self-regulation. European Psychologist, 10, 187-198. doi:10. 1027/1016-9040.10.3.187.

Gendolla, G. H. E., Brinkmann, K., \& Silvestrini, N. (2012). Gloomy and lazy? On the impact of mood and depressive symptoms on effort-related cardiovascular response. In R. A. Wright \& G.
H. E. Gendolla (Eds.), How motivation affects cardiovascular response: Mechanisms and applications (pp. 139-155). Washington, DC: American Psychological Association.

Gendolla, G. H. E., \& Krüsken, J. (2002). Informational mood impact on effort-related cardiovascular response: Moods' diagnostic value counts. Emotion, 2, 251-261. doi:10.1037/1528-3542.2.3. 251.

Gendolla, G. H. E., \& Richter, M. (2010). Effort mobilization when the self is involved: Some lessons from the cardiovascular system. Review of General Psychology, 14, 212-226. doi:10. 1037/a0019742.

Gendolla, G. H. E., \& Silvestrini, N. (2010). The implicit "Go": Masked action cues directly mobilize mental effort. Psychological Science, 21, 1389-1393. doi:10.1177/0956797610384149.

Gendolla, G. H. E., \& Silvestrini, N. (2011). Smiles make it easier and so do frowns: Masked affective stimuli influence mental effort. Emotion, 11, 320-328. doi:10.1037/a0022593.

Gendolla, G. H. E., \& Silvestrini, N. (2015). Bounded effort automaticity: A drama in four parts. In G. H. E. Gendolla, M. Tops, \& S. Koole (Eds.), Handbook of biobehavioral approaches to self-regulation. New York: Springer. doi:10.1007/978-14939-1236-0_18

Herr, P. M. (1986). Consequences of priming: Judgment and behavior. Journal of Personality and Social Psychology, 51, 1106-1115. doi:10.1037/0022-3514.51.6.1106.

Kelsey, R. M. (2012). Beta-adrenergic cardiovascular reactivity and adaptation to stress: The cardiac pre-ejection period as an index of effort. In R. A. Wright \& G. H. E. Gendolla (Eds.), How motivation affects cardiovascular response: Mechanisms and applications (pp. 43-60). Washington, DC: American Psychological Association.

Kreibig, S. D., Gendolla, G. H. E., \& Scherer, K. R. (2012). Goal relevance and goal conduciveness appraisals lead to differential autonomic reactivity in emotional responding to performance feedback. Biological Psychology, 91, 365-375. doi:10.1016/j. biopsycho.2012.08.007.

Lasauskaite, R., Gendolla, G. H. E., \& Silvestrini, N. (2013). Do sadness-primes make me work harder because they make me sad? Cognition and Emotion, 27, 158-165. doi:10.1080/ 02699931.2012 .689756$.

Levick, J. R. (2003). An introduction to cardiovascular physiology (4th ed.). London: Arnold.

Llabre, M. M., Spitzer, S. B., Saab, P. G., Ironson, G. H., \& Schneiderman, N. (1991). The reliability and specificity of delta versus residualized change as measures of cardiovascular reactivity to behavioral challenges. Psychophysiology, 28, 701-711. doi:10.1111/j.1469-8986.1991.tb01017.x.

Locke, E. A., \& Latham, G. P. (1990). A theory of goal setting and task performance. Englewood Cliffs, NJ: Prentice-Hall.

Loersch, C., \& Payne, B. K. (2012). On mental contamination: The role of (mis) attribution in behavior priming. Social Cognition, 30, 241-252. doi:10.1521/soco.2012.30.2.241.

Lozano, D. L., Norman, G., Knox, D., Wood, B. L., Miller, B. D., Emery, C. F., et al. (2007). Where to B in dZ/dt. Psychophysiology, 44, 113-119. doi:10.1111/j.1469-8986.2006.00468.x.

Lundqvist, D., \& Litton, J. E. (1998). The averaged Karolinska directed emotional faces-AKDEF. Stockholm, Sweden: CD ROM from Department of Clinical Neuroscience, Psychology section, Karolinska Institutet.

Matthews, G., Jones, D. M., \& Chamberlain, A. G. (1990). Refining the measurement of mood: The UWIST mood adjective checklist. British Journal of Psychology, 81, 17-42. doi:10.1111/j. 2044-8295.1990.tb02343.x.

Murphy, S. T., \& Zajonc, R. B. (1993). Affect, cognition, and awareness: Affective priming with optimal and suboptimal 
stimulus exposures. Journal of Personality and Social Psychology, 64, 723-739. doi:10.1037/0022-3514.64.5.723.

Obrist, P. A. (1981). Cardiovascular psychophysiology: A perspective. New York: Plenum Press.

Richter, M. (2010). Blue box 2. Geneva, Switzerland: University of Geneva.

Richter, M., Friedrich, A., \& Gendolla, G. H. E. (2008). Task difficulty effects on cardiac activity. Psychophysiology, 45, 869-875. doi:10.1111/j.1469-8986.2008.00688.x.

Robinson, M. D., \& Clore, G. L. (2002). Belief and feeling: Evidence for an accessibility model of emotional self-report. Psychological Bulletin, 128, 934-960. doi:10.1037//0033-2909.128.6.934.

Rotteveel, M., de Groot, P., Geutskens, A., \& Phaf, R. H. (2001). Stronger suboptimal than optimal affective priming? Emotion, 1, 348-364. doi:10.1037//1528-3542.1.4.348.

Ryan, R. M., \& Deci, E. L. (2000). Self-determination theory and the facilitation of intrinsic motivation, social development, and wellbeing. American Psychologist, 55, 78-88. doi:10.1037/0003066X.55.1.68

Scherhag, A., Kaden, J. J., Kentschke, E., Sueselbeck, T., \& Borggrefe, M. (2005). Comparison of impedance cardiography and thermodilution-derived measurements of stroke volume and cardiac output at rest and during exercice testing. Cardiovascular Drugs and Therapy, 19, 141-147. doi:10.1007/s10557-0051048-0.

Sherwood, A., Allen, M. T., Fahrenberg, J., Kelsey, R. M., Lovallo, W. R., \& Doornen, L. J. P. (1990). Methodological guidelines for impedance cardiography. Psychophysiology, 27, 1-23. doi:10. 1111/j.1469-8986.1990.tb02171.x.

Siegel, P., \& Warren, R. (2013). Less is still more: Maintenance of the very brief exposure effect 1 year later. Emotion, 13, 338-344. doi: $10.1037 / \mathrm{a} 003083$.

Siegel, P., \& Weinberger, J. (2012). Less is more: The effects of very brief versus clearly visible exposure. Emotion, 12, 394-402. doi:10.1037/a0026806.

Silvestrini, N., \& Gendolla, G. H. E. (2011a). Do not prime too much: Prime frequency effects of masked affective stimuli on effort- related cardiovascular response. Biological Psychology, 87, 195-199. doi:10.1016/j.biopsycho.2011.01.006.

Silvestrini, N., \& Gendolla, G. H. E. (2011b). Masked affective stimuli moderate task difficulty effects on effort-related cardiovascular response. Psychophysiology, 48, 1157-1164. doi:10. 1111/j.1469-8986.2011.01181.x.

Silvia, P. J. (2012). Mirrors, masks, and motivation: Implicit and explicit self-focused attention influence effort-related cardiovascular reactivity. Biological Psychology, 90, 192-201. doi:10. 1016/j.biopsycho.2012.03.01.

Verwijmeren, T., Karremans, J. C., Bernritter, S. F., Stroebe, W., \& Wigboldus, D. H. (2013). Warning: You are being primed! The effect of a warning on the impact of subliminal ads. Journal of Experimental Social Psychology, 49, 1124-1129. doi:10.1016/j. jesp.2013.06.01.

Winkielman, P., Berridge, K. C., \& Wilbarger, J. L. (2005). Emotion, behavior, and conscious experience. In L. F. Barrett, P. M. Niedenthal, \& P. Winkielman (Eds.), Emotion and consciousness (pp. 334-362). New York: Guilford.

Winkielman, P., \& Schooler, J. W. (2011). Splitting consciousness: Unconscious, conscious, and metaconscious processes in social cognition. European Review of Social Psychology, 22, 1-35. doi:10.1080/10463283.2011.57658.

Wright, R. A. (1996). Brehm's theory of motivation as a model of effort and cardiovascular response. In P. M. Gollwitzer \& J. A. Bargh (Eds.), The psychology of action (pp. 424-453). New York: Guilford Press.

Wright, R. A. (1998). Ability perception and cardiovascular response to behavioral challenge. In M. Kofta, G. Weary, \& G. Sedek (Eds.), Personal control in action: Cognitive and motivational mechanisms (pp. 197-232). New York: Plenum Press.

Wright, R. A., \& Kirby, L. D. (2001). Effort determination of cardiovascular response: An integrative analysis with applications in social psychology. Advances in Experimental Social Psychology, 33, 255-307. doi:10.1016/S0065-2601(01)80007-1. 